\title{
New Possibilities for State-of-the-Art Electron Microscopy with Fast Backscattered Electron Detectors
}

Maximilian Schmid ${ }^{1}$, Andreas Liebel ${ }^{1}$, Grigore Moldovan ${ }^{2}$, Robert Lackner ${ }^{1}$, Daniel Steigenhöfer ${ }^{1}$, Adrian Niculae ${ }^{1}$, Heike Soltau ${ }^{1}$

1. PNDetector GmbH, Otto-Hahn-Ring 6, München, Germany

2. point electronic GmbH, Erich-Neuß-Weg 15, Halle (Saale), Germany

Today's Scanning Electron Microscopes are aiming for fast sample processing from sample insertion to the final picture or information needed. TV speed imaging at high resolutions helps to search and identify sample regions as well as to acquire high quality pictures without the need for a search in reduced image resolution mode. However, not all types of detectors are able to support the capabilities offered by new SEMs. For compositional examination, Secondary Electron (SE) detectors are often used for coarse searching before switching to mostly slower Backscattered Electron (BSE) detectors. This costs time and leads to superfluous electron exposure to the specimen. Furthermore, the two different detector types provide different sample information, and thus hinder finding the region of interest on the specimen. In this paper, we show the advantages of exclusively using BSE detection with a high speed segmented BSD module in the context of work flow and fast image acquisition.

To support the high speeds of the BSE-Chip, the diode and the preamplifier board form one unit placed in a flat high-grade housing. Figure 1 shows the BSE detector module. Equipped with a BSE diode with $1 \mathrm{~mm}$ inner hole and $40 \mathrm{~mm}^{2}$ sensitive area, the module exhibits a maximum Geometric Collection Efficiency (GCE) of $50 \%$ at a distance of $2 \mathrm{~mm}$ between the sample and diode. Such high GCE enable high signal outputs even at low beam currents. The close connection of the BSE-Diode to the preamplifier board ensures minimum stray capacitance between signal generation and amplification. In addition, the design of the amplifier is geared to the diode, which enables the BSE detector rise times down to $20 \mathrm{~ns}$, at an amplification factor of $10^{5} \mathrm{~V} / \mathrm{A}$. Figure 2 shows the step response of the BSE detector module to a fast switching light pulse. As an example full HD video (1920x1080 pixel) can be captured at a frame rate of $24 \mathrm{~Hz}$, highlighting the outstanding speed of the BSE detector module and the minimal interaction time between the electron beam and the specimen.

The advantage of fast BSE detection becomes obvious when comparing it to standard measuring procedures. The switching between SE and BSE detectors for coarse adjustment and information collection can be omitted leading to significant improvement in work flow, results and time consumption. Furthermore, the ability to collect and process the signals of four individual quadrants simultaneously ensures distinction of topographical information from compositional contrast. Figure 3 shows the live view of a structured Al film on a Si wafer taken at a pixel rate of $50 \mathrm{Mhz}$ ( $20 \mathrm{~ns}$ pixel dwell time). Next to the compositional and topographic images, the real time topographic surface reconstruction offers height information and helps to understand the sample properties, completing the overall view. In addition, shorter timescales ensure a minimization of electron exposure of the specimen. This becomes especially important when analyzing electron sensitive materials as in life science or serial block-face imaging. There, information has to be collected quickly and reliably with minimal exposure of the specimen to the electron beam. Fast BSE detectors can be used in a wide variety of applications without being limited in speed and overview, even in 
challenging scanning conditions.

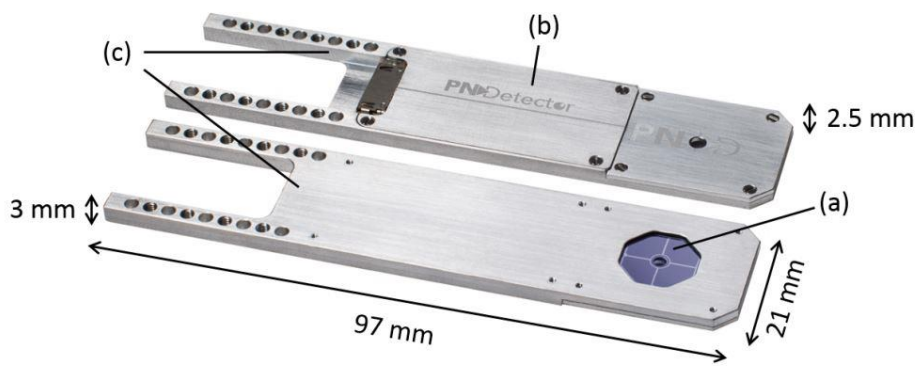

Figure 1. Front and backside view of the high speed BSE detector module: (a) 4 Quadrant BSE Diode, (b) 4 channel preamplifier, directly connected to the diode, (c) ultra flat aluminum housing to fit below the pole piece.

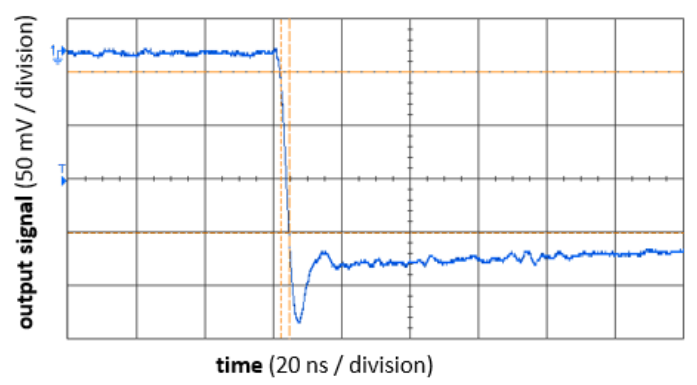

Figure 2. BSE detector module output vs. time. Signal rise times of about 20 to $25 \mathrm{nsec}$ are measured by illuminating the BSE-detector with a high speed LED pulser with a switch on rise time of $7 \mathrm{~ns}$.
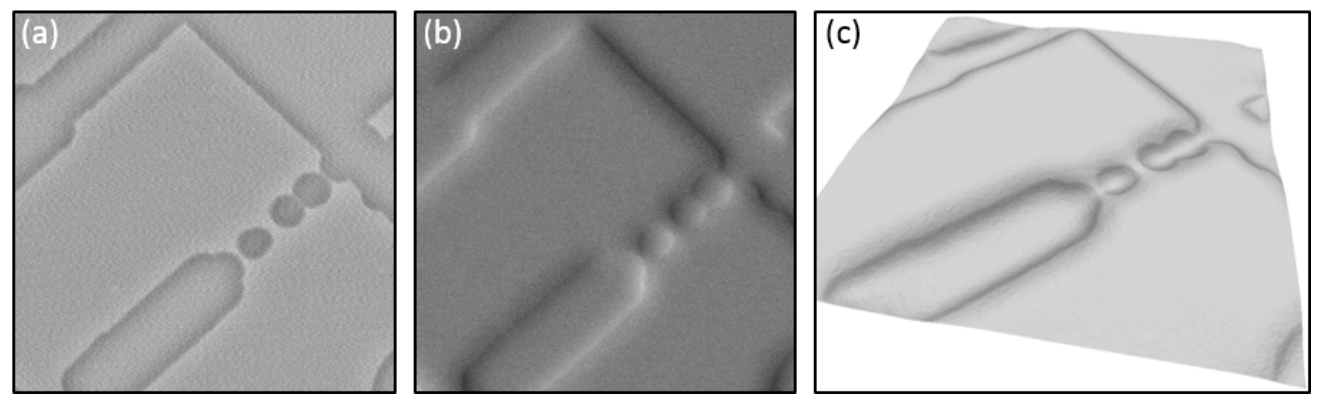

Figure 3. Live view of a structured Al layer on a Si wafer. Images obtained simultaneously at a scan speed of $20 \mathrm{~ns}$ pixel dwell time using an ultra fast scan and acquisition system with dedicated surface reconstruction software. (a) Compositional contrast. (b) Topographical contrast. (c) Real time topographic surface reconstruction. 